\title{
Rho-rho production in two-photon collisions
}

\author{
Klaus Freudenreich ${ }^{* \dagger}$ \\ Labor für Hochenergiephysik, ETH Zürich, Switzerland \\ E-mail: Klaus.Freudenreichecern.ch
}

The measurement of exclusive $\rho \rho$ production in two-photon interactions at LEP, $\gamma \gamma^{*} \rightarrow \rho \rho$, was studied at two-photon center-of-mass energies of $1.1 \mathrm{GeV} \leq \mathrm{W}_{\gamma \gamma} \leq 3 \mathrm{GeV}$ and photon virtualities of $\mathrm{Q}^{2}<0.02 \mathrm{GeV}^{2}$ and $0.2 \leq \mathrm{Q}^{2} \leq 30 \mathrm{GeV}^{2}$. These data allow on the one hand a comparison to QCD and the generalised vector dominance model (GVDM). On the other hand, the large kinematical range permits to check models with exotic mesons.

International Europhysics Conference on High Energy Physics

July 21st - 27th 2005

Lisboa, Portugal

* Speaker.
$\dagger$ On behalf of the L3 collaboration. 


\section{Introduction}

The two-photon process

$$
e^{+} e^{-} \rightarrow e^{+} e^{-} \gamma \gamma^{*} \rightarrow e^{+} e^{-} \rho \rho
$$

has already been measured at lower $e^{+} e^{-}$c.m. energies [1] but mostly without tagging. The data presented here were obtained with tagging, thus allowing to cover a larger range in the virtuality of one of the interacting photons, i.e. $0.2 \leq \mathrm{Q}^{2} \leq 30 \mathrm{GeV}^{2}$. For comparison, also preliminary untagged data at $\mathrm{Q}^{2}<0.02 \mathrm{GeV}^{2}$ will be shown. Both $\rho^{0} \rho^{0} \rightarrow \pi^{+} \pi^{-} \pi^{+} \pi^{-}$and $\rho^{+} \rho^{-} \rightarrow \pi^{+} \pi^{0} \pi^{-} \pi^{0}$ channels are studied. The large range in the two-photon c.m. energy, $1.1 \mathrm{GeV} \leq \mathrm{W}_{\gamma \gamma} \leq 3 \mathrm{GeV}$ allows to study resonance production in t-channel exchange as well as to search for exotic mesons.

\section{Data}

The data were taken with the L3 detector [2] at LEP (Fig. 1).

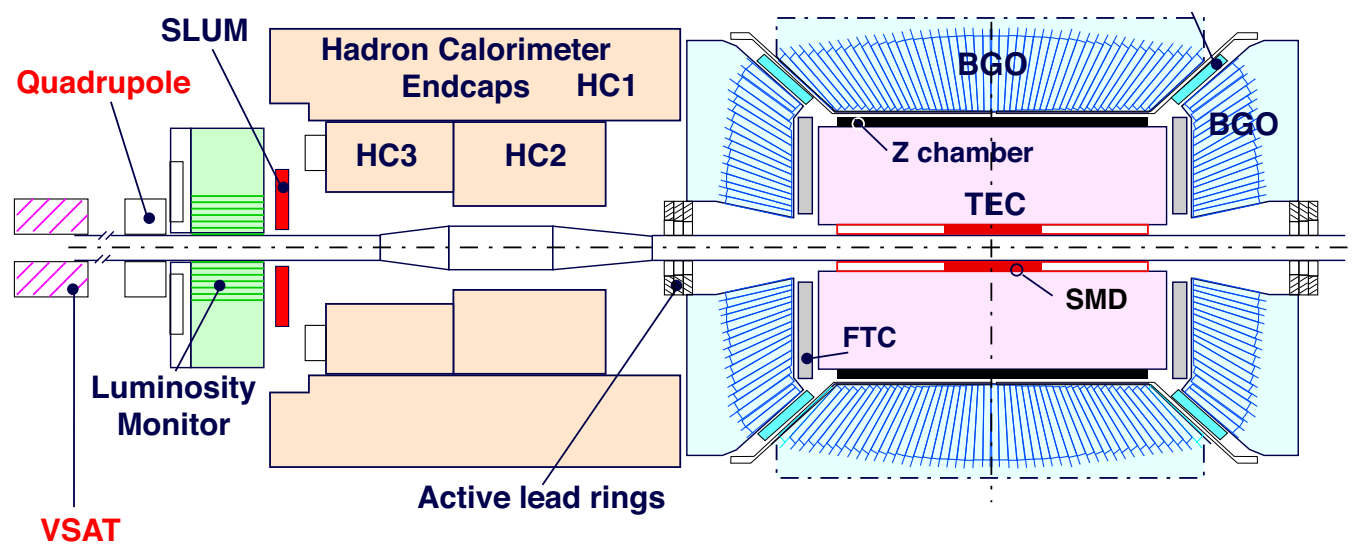

Figure 1: Sketch (sideview) of the L3 detectors used in this analysis.

The L3 detector was well suited for this search because there was only a small amount of material in front of the electromagnetic BGO calorimeter ( 0.2 of a radiation length). This yielded a low threshold in photon energies $(\geq 60 \mathrm{MeV})$ and in momentum measurements of charged tracks $\left(\mathrm{p}_{T} \geq 100 \mathrm{MeV}\right)$. Two detectors were used for tagging: a) The so called very small angle tagger (VSAT) detectors, situated on either side of the interaction point (IP) at a distance of $8.17 \mathrm{~m}$, behind the first quadrupole. It consisted of 4 BGO crystal calorimeters. b) The luminosity monitors situated at either side of the IP at a distance of $2.73 \mathrm{~m}$, each consisting of 2 detectors with 304 BGO crystals per detector.

In the following, the data will be divided into four intervals in $\mathrm{Q}^{2}$ :

1. $\mathrm{Q}^{2}<0.02 \mathrm{GeV}^{2}$, no electron tag, $\mathrm{Q}^{2}$ calculation from the $4 \pi$ state,

2. $0.2 \mathrm{GeV}^{2}<\mathrm{Q}^{2}<0.85 \mathrm{GeV}^{2}$, electron tag from the VSAT, $\mathrm{Q}^{2}$ calculation from the $4 \pi$ state,

3. 1.2 $\mathrm{GeV}^{2}<\mathrm{Q}^{2}<8.5 \mathrm{GeV}^{2}$ electron tag and $\mathrm{Q}^{2}$ calculation from the luminosity monitor,

4. 8.8 $\mathrm{GeV}^{2}<\mathrm{Q}^{2}<30 \mathrm{GeV}^{2}$, electron tag and $\mathrm{Q}^{2}$ calculation from the luminosity monitor. 
The tagged data were taken at $91 \mathrm{GeV} \leq \sqrt{s} \leq 209 \mathrm{GeV}$ with an integrated luminosity of $854.7 \mathrm{pb}^{-1}$ [3]. The untagged data were taken at $161 \mathrm{GeV} \leq \sqrt{s} \leq 209 \mathrm{GeV}$ with an integrated luminosity of $697.7 \mathrm{pb}^{-1}$ [4].

Fig. 2 shows the four-pion mass distribution $\left(\mathrm{W}_{\gamma \gamma}\right)$ and the 4 possible mass combinations $\mathrm{M}\left(\pi^{ \pm} \pi^{0}\right)$ (within $1.1 \mathrm{GeV} \leq W_{\gamma \gamma} \leq 3 \mathrm{GeV}$ ) for the reaction $e^{+} e^{-} \rightarrow e^{+} e_{\text {tag }}^{-} \pi^{+} \pi^{-} \pi^{0} \pi^{0}$ for 0.2 $\mathrm{GeV}^{2}<\mathrm{Q}^{2}<0.85 \mathrm{GeV}^{2}$. A clear $\rho^{ \pm}$signal is observed in $\mathrm{M}\left(\pi^{ \pm} \pi^{0}\right)$.
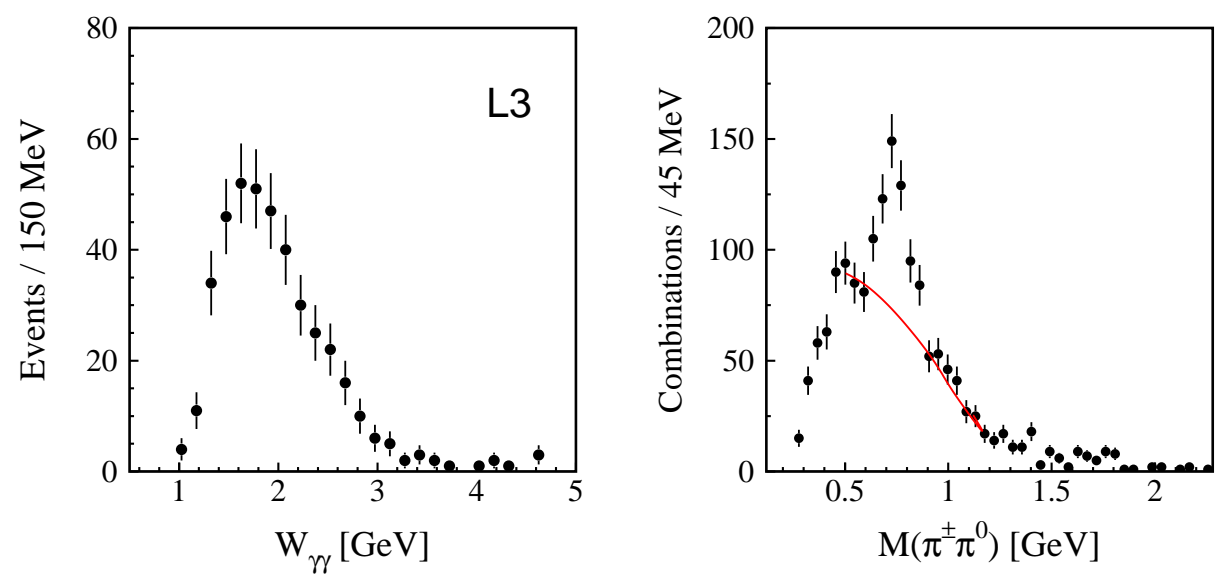

Figure 2: $\mathrm{M}\left(\pi^{+} \pi^{-} \pi^{0} \pi^{0}\right)$ (left) and $\mathrm{M}\left(\pi^{ \pm} \pi^{0}\right), 4$ entries per event (right).

Fig. 3 shows the 4 pion invariant mass distribution $\left(\mathrm{W}_{\gamma \gamma}\right)$ and the 4 possible mass combinations $\mathrm{M}\left(\pi^{+} \pi^{-}\right)$(within $1.1 \mathrm{GeV} \leq W_{\gamma \gamma} \leq 3 \mathrm{GeV}$ ) for the reaction $e^{+} e^{-} \rightarrow e^{+} e_{\text {tag }}^{-} \pi^{+} \pi^{-} \pi^{+} \pi^{-}$for 0.2 $\mathrm{GeV}^{2}<\mathrm{Q}^{2}<0.85 \mathrm{GeV}^{2}$. A strong $\rho^{0}$ signal is observed in $\mathrm{M}\left(\pi^{+} \pi^{-}\right)$.
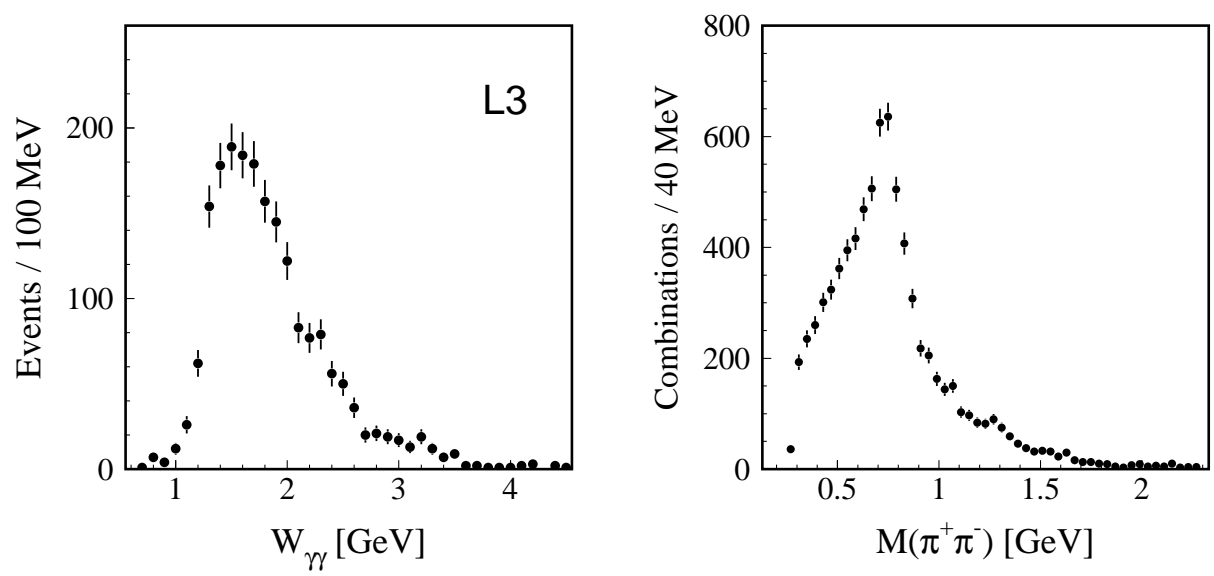

Figure 3: $\mathrm{M}\left(\pi^{+} \pi^{-} \pi^{+} \pi^{-}\right)$(left) and $\mathrm{M}\left(\pi^{+} \pi^{-}\right), 4$ entries per event (right).

The fraction of $\rho \rho$ events was determined by a maximum likelihood fit in intervals of $\mathrm{Q}^{2}$ and $\mathrm{W}_{\gamma \gamma}$. For the background, the following processes were considered: $\gamma \gamma^{*} \rightarrow \rho \pi \pi, \gamma \gamma^{*} \rightarrow$ $a_{2}^{ \pm}(1320) \pi^{\mp}, \gamma \gamma^{*} \rightarrow f_{2} \pi \pi$ and nonresonant $\gamma \gamma^{*} \rightarrow \pi \pi \pi \pi$. 
The cross section $\sigma_{\gamma \gamma}\left(\gamma \gamma^{*} \rightarrow \rho \rho\right)$ was obtained from the the $\sigma_{e e}\left(e^{+} e^{-} \rightarrow e^{+} e^{-} \rho \rho\right)$ cross section via $\sigma_{e e}=L_{T T} \cdot \sigma_{\gamma \gamma}$ where $L_{T T}$ is the two-photon luminosity function which is calculated using the program GALUGA [5].

\section{Results}

Fig. 4 shows the $\gamma \gamma \rightarrow \rho^{0} \rho^{0}$ and $\gamma \gamma \rightarrow \rho^{+} \rho^{-}$cross sections as a function of the four-pion masses in the four $\mathrm{Q}^{2}$ intervals mentioned above.
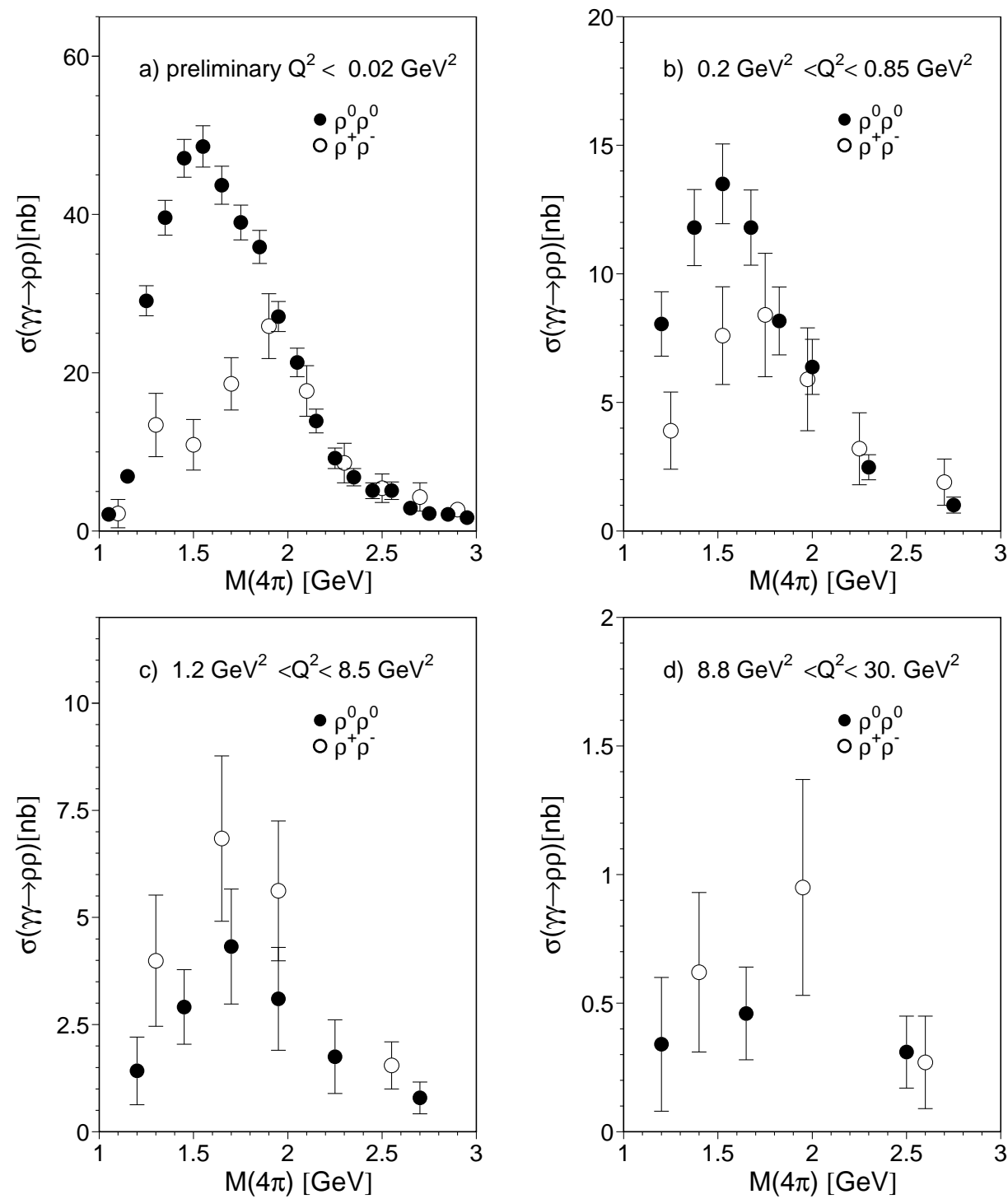

Figure 4: The $\gamma \gamma \rightarrow \rho^{0} \rho^{0}$ and $\gamma \gamma \rightarrow \rho^{+} \rho^{-}$cross sections as a function of the four-pion mass a) at $Q^{2} \leq$ $0.02 \mathrm{GeV}^{2}$, b) $0.20 \mathrm{GeV}^{2} \leq Q^{2} \leq 0.85 \mathrm{GeV}^{2}$, c) $1.2 \mathrm{GeV}^{2} \leq Q^{2} \leq 8.5 \mathrm{GeV}^{2}$ and d) $8.8 \mathrm{GeV}^{2} \leq Q^{2} \leq 30 \mathrm{GeV}^{2}$.

For an isospin $\mathrm{I}=0$ state the ratio $\mathrm{R}$ of $\sigma\left(\gamma \gamma \rightarrow \rho^{+} \rho^{-}\right) / \sigma\left(\gamma \gamma \rightarrow \rho^{0} \rho^{0}\right)$ is equal two. We observe this for $\mathrm{Q}^{2}>1.2 \mathrm{GeV}^{2}$. At low $\mathrm{Q}^{2}$, however, this ratio is reversed to $\mathrm{R}=0.42 \pm 0.05 \pm$ 0.09 for $\mathrm{Q}^{2}<0.02 \mathrm{GeV}^{2}$. This strong enhancement of $\rho^{0} \rho^{0}$ with respect to $\rho^{+} \rho^{-}$at the lowest $\mathrm{Q}^{2}$ value was interpreted as evidence for an isospin 2 resonance [6]. 
In Fig. 5 the cross section $d \sigma_{e e} / d Q^{2}$ is compared to a QCD-based calculation [7] and the $\sigma_{\gamma \gamma}$ cross section is compared to a parametrisation based on the GVDM model [8]. The QCD parametrisation fits both $\sigma\left(\rho^{+} \rho^{-}\right)$and $\sigma\left(\rho^{0} \rho^{0}\right)$ well over four orders of magnitude. There is a crossover of the cross sections at a $\mathrm{Q}^{2}$ of around $1 \mathrm{GeV}^{2}$ suggesting a different production mechanism at low and high $\mathrm{Q}^{2}$. The GVDM parametrisation reproduces only $\sigma\left(\rho^{0} \rho^{0}\right)$ well. The $\mathrm{Q}^{2}$ evolution of $\sigma\left(\rho^{+} \rho^{-}\right)$cannot be described by this parametrisation. A $\rho$-pole fit to the data fails for both the $\rho^{0} \rho^{0}$ and the $\rho^{+} \rho^{-}$cross sections.
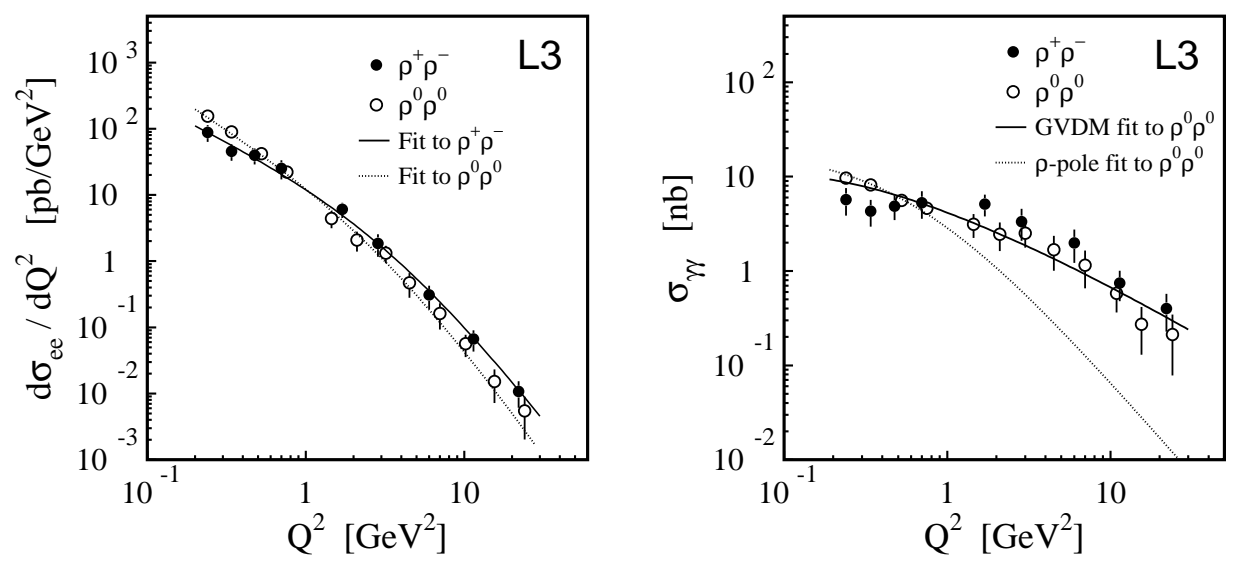

Figure 5: $\frac{d \sigma_{e e}}{d Q^{2}}$ (left) and $\sigma_{\gamma \gamma}$ (right) as a function of $\mathrm{Q}^{2}$.

\section{Acknowledgements}

I would like to thank my colleagues from the L3 Two-Photon group for their advice: J. Field, M. Kienzle, G. Sultanov and I. Vorobiev.

\section{References}

[1] M. Althoff et al., Z. Phys. C 16 (1982) 13; Ch. Berger et al., Z. Phys. C 38 (1988) 521; W. Braunschweig et al., Z. Phys. C 41 (1988) 353; H. Albrecht et al., Z. Phys. C 50 (1991) 1; H. Albrecht et al., Phys. Lett. B 267 (1991) 535.

[2] B. Adeva et al., Nucl. Instr. and Methods A 289 (1990) 35; M. Chemarin et al., Nucl. Instr. and Methods A 349 (1994) 345; M. Acciarri et al., Nucl. Instr. and Methods A 351 (1994) 300; I.C. Brock et al., Nucl. Instr. and Methods A 381 (1996) 236; A. Adam et al., Nucl. Instr. and Methods A 383 (1996) 342.

[3] P. Achard et al., Phys. Lett. B 568 (2003) 11, P. Achard et al., Phys. Lett. B 597 (2004) 26, P. Achard et al., Phys. Lett. B 604 (2003) 48, P. Achard et al., Phys. Lett. B 615 (2005) 19.

[4] L3 Coll., in preparation.

[5] G.A. Schuler, Comp. Science Commun. 108 (1998) 279.

[6] I.V. Anikin, B. Pire and O.V. Teryaev, Phys. Lett. B 626 (2005) 86.

[7] M. Diehl, T. Gousset and B. Piere, Phys. Rev. D 62 (2000) 073014.

[8] J.J. Sakurai and D. Schildknecht, Phys. Lett. B 40 (1972) 121; I.F. Ginzburg and V.G. Serbo, Phys. Lett. B 109 (1982) 231. 